\title{
ANÁLISE DE IMAGENS TERMOGRÁFICAS PARA DETECÇÃO PRECOCE DE NEOPLASIAS (TUMOR DE MAMA)
}

\author{
Thais Gretis Rodrigues da Luz ${ }^{1}$, José Carlos Coninck ${ }^{2}$, Leandra Ulbricht ${ }^{3}$
}

Programa de Pós Graduação em Engenharia Biomédica - PPGEB /UTFPR

2 Departamento Acadêmico de Estatística - DAEST /UTFPR

3 Programa de Pós Graduação em Engenharia Biomédica - PPGEB /UTFPR

Resumo: A termografia é um método não invasivo, sem contato físico, não apresentando riscos para o paciente ou para o aplicador. Os objetivos deste estudo foram identificar protocolos para: a) verificar os protocolos que tiveram resultados positivos para o uso da termografia. b) identificar os pontos em comum entre eles. Este é um estudo exploratório que utilizou como ferramenta a bibliometria, sendo incluídos os artigos publicados entre 2012 a 2018. Foi realizada uma análise descritiva onde os artigos foram categorizados, e descritos conforme os dados de publicação, autoria e equipamento utilizado. Os resultados apontam uma variedade de protocolos de utilização da termografia no estudo do auxílio ao diagnóstico de câncer de mama. Como conclusão identifica-se resultados positivos para o uso da termografia na confirmação do câncer de mama. Sendo que, a maior parte destes estudos utilizou os seguintes critérios: Deve-se manter a sala entre $22^{\circ} \mathrm{C}$ e $23^{\circ} \mathrm{C}$ (graus Celsius), realizar uma aclimatação da paciente, uso de câmeras com melhores resoluções (maiores que $160 \times 120$ pixels) e uso do protocolo dinâmico, para melhor ressaltar as diferenças térmicas entre os tecidos mamários sadios e tumorais.

Palavras-chave: Termografia; Câncer de mama; Protocolo

\begin{abstract}
Thermography is a noninvasive method, without physical contact, presenting no risk to the patient or the applicator. The objectives of this study were to identify protocols to: a) verify the protocols that had positive results for the use of thermography. b) identify the commonalities between them. This is an exploratory study that uses as a bibliometrics as a tool, being including articles published between 2012 and 2018. A descriptive analysis was performed where the articles were categorized, and described according to the publication data, authorship and equipment used. The results point to a series of protocols for the use of thermography in the confirmation diagnosis of breast cancer. In conclusion, positive results were identified for the use of thermography in the verification of breast cancer. Most of these items used are as follows: A room should be kept between $22^{\circ} \mathrm{C}$ and $23^{\circ} \mathrm{C}$ (degrees Celsius), perform a patient acclimatization, use cameras with better measurements (larger than $160 \times 120$ pixels) and use the dynamic protocol to better highlight the thermal differences between breast tissue and tumor tissues..
\end{abstract}

Keywords: Thermography; Breast cancer; Protocol 


\section{PAN AMERICAN JOURNAL OF MEDICAL THERMOLOGY}

\section{INTRODUÇÃO}

A neoplasia mamária é o mais prevalente tipo de câncer entre as mulheres no Brasil, de acordo com o Instituto Nacional de Câncer (INCA). Aponta-se a incidência para 59.700 novos casos no ano de 2018/2019.

Essa doença se caracteriza pelo crescimento de células anormais, sendo estas, células neoplásicas que multiplicam-se desordenadamente em órgãos e tecidos, podendo se espalhar para diferentes regiões do corpo, caracterizando metástase. ${ }^{1}$

Estudos afirmam que em neoplasias diagnosticadas em estágio inicial eleva-se para $85 \%$ as chances de cura e, quando em estágios avançados, cai para 10\%.; 4

Como formas de diagnóstico, pode-se utilizar o autoexame ou o monitoramento por imagem, como a mamografia, ultrassonografia, ressonância magnética e tomossíntese. ${ }^{5 ;} 6$

Essas técnicas foram desenvolvidas como métodos clínicos primários, que visam identificar neoplasias de mama em estágios iniciais, ou diferenciá-los de benignos/malignos ou multicêntricos, e acompanhar o tratamento.

No entanto, esses métodos apresentam limitações. A mamografia, por exemplo, método indicado no diagnóstico de avaliação das mamas, é um procedimento desconfortável, que apresenta menor sensibilidade para mulheres com tecido mamário denso, alto percentual de falsos positivos e emissão de radiação ${ }^{2 ; 6 ; 7 ; 8 ; 9}$

O ultrassom de mama, a tomografia e a ressonância magnética são recomendados para confirmação de presença ou ausência de nódulos, ou diminuição de falsos positivos da MMG, mas apresentam baixa sensibilidade para múltiplos focos malignos e microcalcificações. Além disso, são procedimentos de alto custo, exigem maior conhecimento técnico específico e mão de obra. ${ }^{2 ;} 8 ; 10 ; 11 ; 12$

Alguns estudos tem investigado o potencial da termografia para ser utilizada como método diagnóstico complementar no monitoramento por imagem. ${ }^{13 ;} 14 ; 15 ; 16$ A maior parte da literatura tem apresentado a termografia com características e resultados promissores. ${ }^{15 ; 17 ; 18}$ Contudo, os estudos ainda apresentam uma enorme heterogeneidade nas condições de coleta, tecnologias, e preparação do paciente. Essas condições podem interferir nos resultados e consequentemente, na viabilidade do método.

O estudo apresentado busca na literatura, um protocolo que apresente bons resultados, quanto ao uso da termografia para possível diagnóstico de câncer de mama, para embasar assim uma melhor consistência de pesquisas futuras.

\section{METODOLOGIA}

Foi utilizado um portfólio de pesquisas sobre o tema e conduzido uma análise bibliométrica, para que com isso fosse possível investigar particularidades da produção científica, referentes ao progresso nos resultados das pesquisas.

Procedeu-se a pesquisa quantitativa, de caráter exploratório e descritivo, com pesquisas relacionadas a termografia e câncer de mama, avaliando os critérios utilizados. As pesquisas estavam dispostas, com acesso permitido na Base de dados disponibilizada pela CAPES - Coordenação de Aperfeiçoamento de Pessoal de Nível Superior.

A busca envolveu pesquisas publicadas no período de 2012 a 2018 nas seguintes bases de dados: Web of Science, Scopus e Scielo. Os algoritmos utilizados foram: Termografia AND Câncer de mama; Thermography AND Diagnosis AND Breast Cancer; Infrared thermography AND Breast cancer; com as seguintes palavras-chave de ex- 
clusão: animal; others câncer pathologies, para identificação de artigos relacionados e publicados.

Essa seleção ocorreu conforme a Figura 1 , sendo que a etapa de seleção inicial da pesquisa, resultou em um total de 148 documentos, que atenderam aos algoritmos. Em seguida, passaram pelos seguintes filtros: exclusão dos títulos repetidos e redundantes, exclusão dos que continham apenas uma das palavras-chave selecionadas e exclusão dos artigos que não estavam completos na base de dados.

Posteriormente, realizou-se leitura dos resumos dos artigos restantes e exclusão dos que não apresentavam delineamento do tema correspondente ao objetivo da pesquisa, e por último leitura dos artigos restantes na íntegra.

Foram localizados ao todo 73 documentos, onde ainda foram excluídos os artigos que analisavam apenas algoritmos e métodos estatísticos para resultados finais, ou que utilizaram base de dados de imagens. O portfólio bibliográfico contou com 23 artigos com as pesquisas que estruturaram todo o processo de aquisição de imagens, coleta com pacientes, condições do ambiente e resultados.

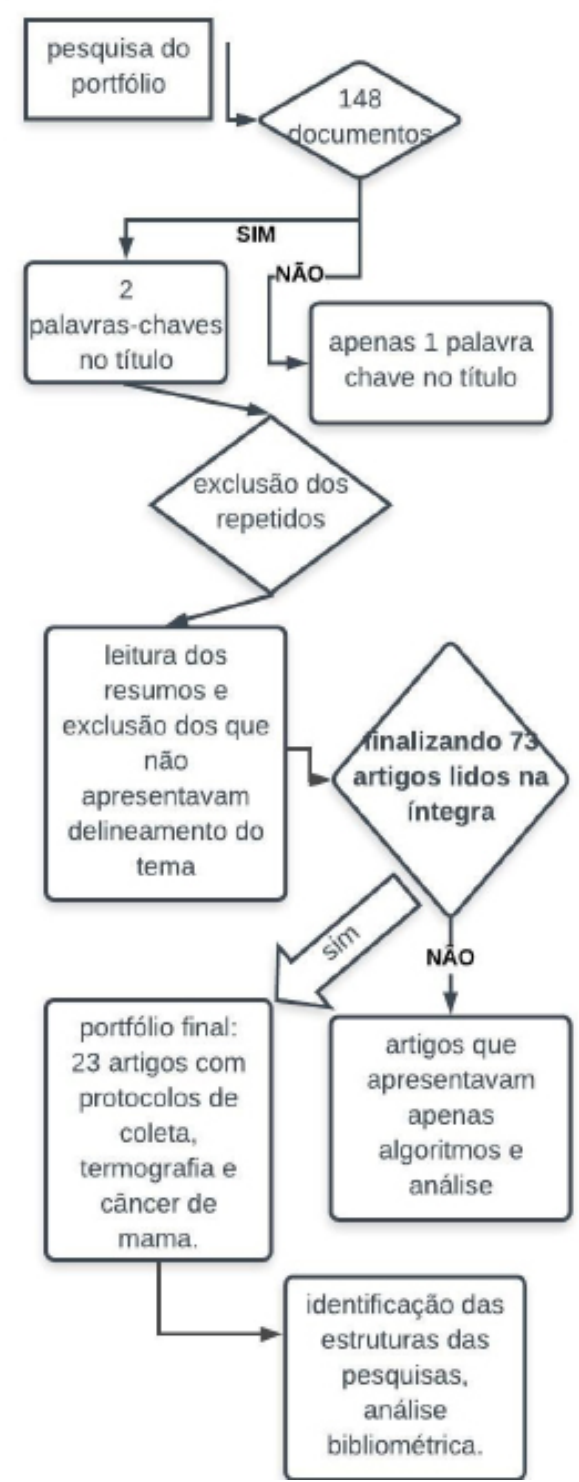

Figura 1: Metodologia utilizada para esco-Iha do portfólio. 


\section{RESULTADOS}

Observa-se na Figura 2 a distribuição dos documentos publicados no período de 2012 a 2018, compreendendo uma evolução das publicações ao longo dos anos, com maior produção científica no ano de 2015 , com $26,08 \%$ das publicações selecionadas.

As pesquisas e publicações ocorreram em 9 países ao todo, com destaque para o Brasil e EUA, com 21,7\% de obras publicadas (Figura 3).

As revistas com mais publicações dentre os artigos selecionados foram o Journal Medical Systens e a Infrared Physics Thecnology com $13,04 \%$ das publicações (Figura 4). A Journal Medical of Systens é uma revista que apresenta discussões sobre novas técnicas e métodos de sistemas no âmbito da saúde, com foco em processamento de imagens e qualidade de sistemas, e fator de impacto de 2.456. A Infrared Physics Thecnology conta com publicações e pesquisas voltadas para todo o campo da física e tecnolo-

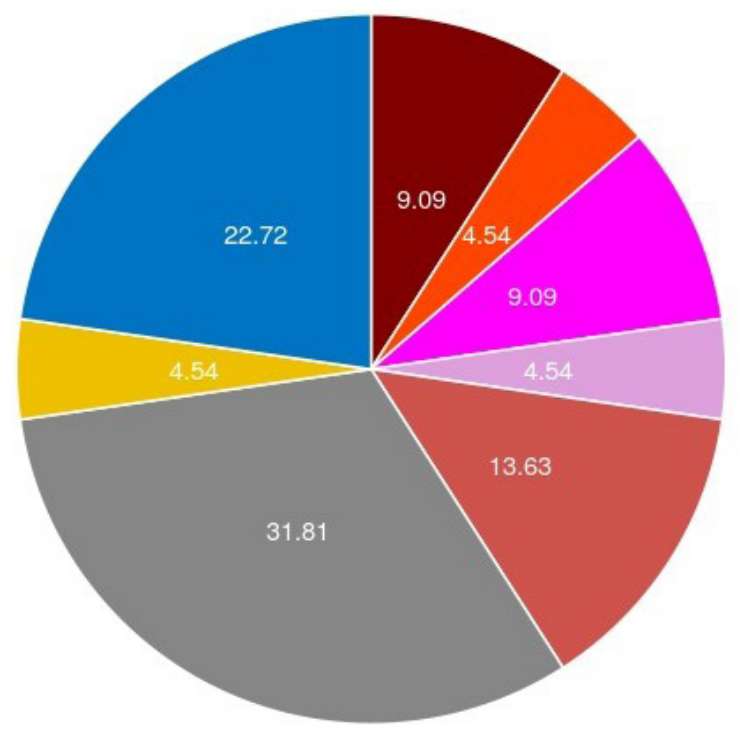

Figura 3 - Distribuição de publicações por países

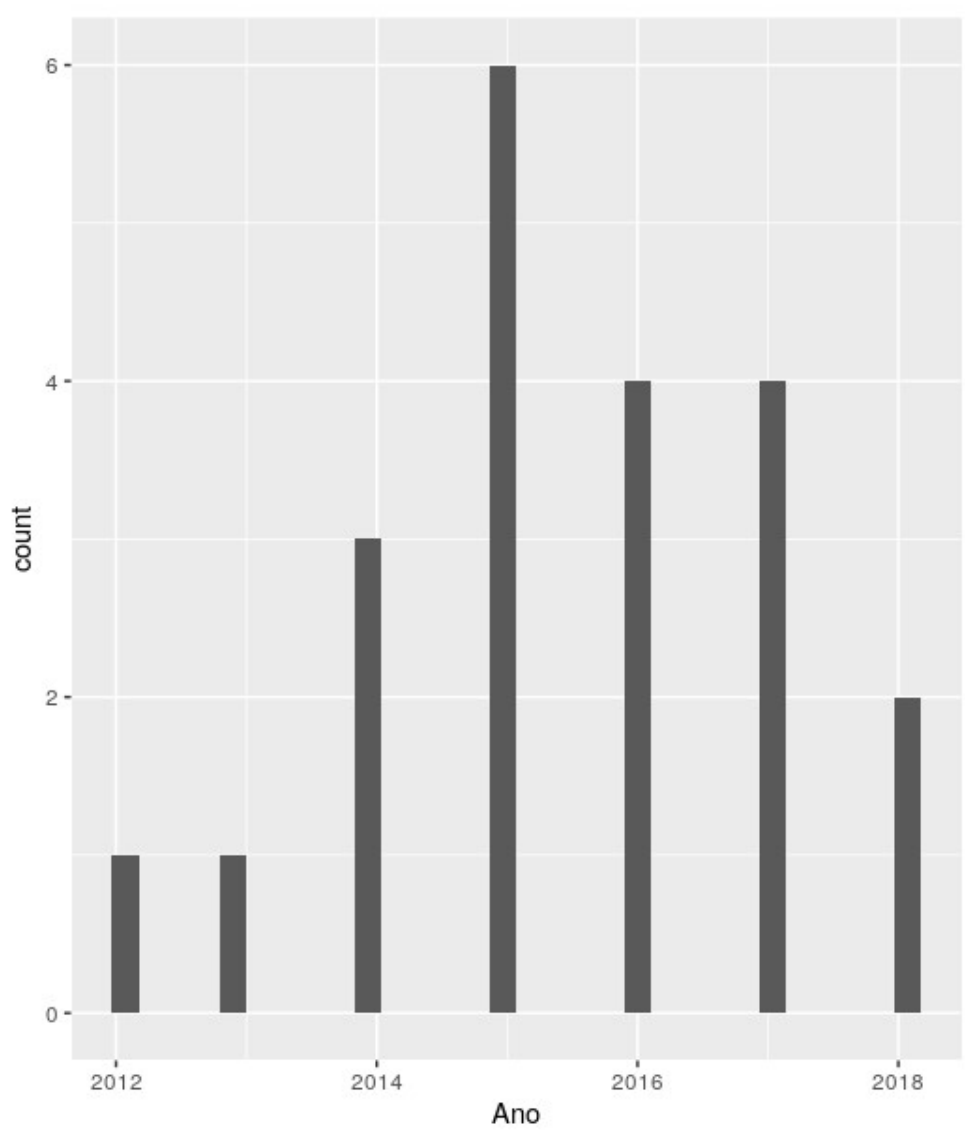

Figura 2 - Distribuição dos artigos por ano

gia de infravermelho: teoria, experimento, aplicação, dispositivos e instrumentação. Voltados para o uso no âmbito da saúde e avanços tecnológicos, seu fator de impacto é de 1.851. Concentrando 8,69\% de publicações estão as revistas: Breast Care, Diangnosis Radiology, Sensors, IEEE e EXCLI Journal

Uma análise realizada neste presente estudo constatou os autores que se destacaram com uma maior trajetória nas pes- class

Brasll

Croácla

EUA

Indla

Ira

México

Sérvia

Singapura quisas relacionadas a termografia e câncer de mama, esta informação torna-se relevante devido a indicação de pesquisadores que devem ser buscados por apresentarem em seu currículo estudos e atualizações sobre o tema em questão.

Como resultados os seguintes autores foram identificados: Eddie YK. NG (14 artigos)², Ma- 
nhaz Etehadtavakol (8 artigos) ${ }^{19}$, Lincoln F. Silva ${ }^{18}$ e U. Rajendra Acharya (4 artigos) ${ }^{20}$, eles se destacaram entre os outros autores porque produziram mais artigos e foram mencionados como referências bibliográficas nos outros artigos do portfólio.

Os seguintes autores também podem ser identificados com participações: Svetlana Antonini18; Marcus C. Araújo ${ }^{21}$; KhritikaVenkataramani22; Sheeva V. Francis $^{23}$, com três estudos cada.

Dos 23 artigos analisados, 95,65\% apresentaram comparação entre os métodos de imagem utilizados para triagem e diagnóstico de câncer de mama, como a mamografia, ultrassom e biópsia. Além disso, utilizaram os resultados dos mesmos para comparação de sensibilidade, especificidade e precisão da termografia. As características dos grupos analisados se mantêm entre 40 e 75 anos de idade, sendo um público estritamente feminino.

Estes artigos, apresentaram resultados positivos para a termografia, e estatisticamente próximos aos da MMG, US e biópsia. 4 ; $20 ; 18 ; 24 ; 25 ; 10 ; 22 ; 16 ; 26 ; 27 ; 28 ; 15$

Rassiwala et al $(2017)^{7}$, Raghavendra et al (2016) ${ }^{3}$ e Venkataramani et al (2015) 22, apresentaram resultados próximos a 100\% (97,06\%; 96,66\%; 94\% de sensibilidade e $99,17 \% ; 100 \%$; $97 \%$ de especificidade, respectivamente) para análises diagnóstico de câncer e de malignidade.

Cerca de 30,43\% dos artigos apresentaram média de $85 \%$ entre os resultados de sensibilidade e especificidade ${ }^{17 ;}$ 29; 14; 30; 10; 31; ${ }^{20}$. E apenas 13,04\% apresentaram resultados próximos a $50 \%$, no quesito especificidade. As pesquisas de Collet et al (2014) ${ }^{27}$ e Jesus de Guirro et al (2017) ${ }^{8}$ utilizaram a comparação de simetrias entre as mamas, o que pode justificar os resultados abaixo da média apresentada pelas pesquisas.

A pesquisa de NG et al.

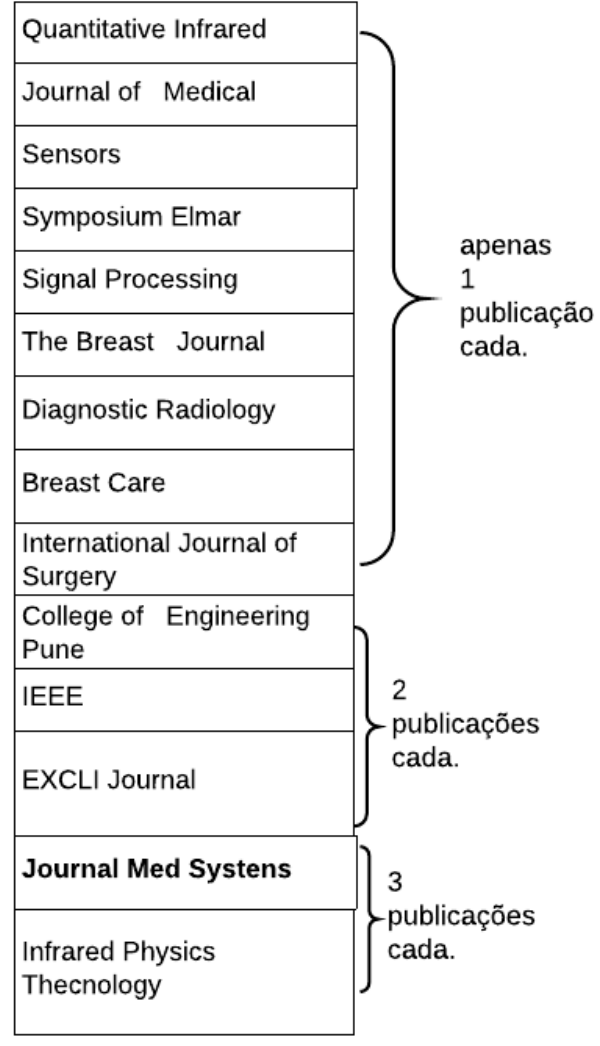

Figura 4 - Número de publicações por revistas

apresentou tanto a especificidade quanto a sensibilidade com médias mais baixas dentre as pesquisas analisadas. Sendo que esta pesquisa foi realizada com o maior número de pacientes com anormalidades benignas na região da mama e análise de temperatura em pacientes sem anormalidades, comprovadas em MMG, US e biópsias, contando com apenas 4 casos de malignidade, em seu grupo de análise, o que pode ter influenciado diretamente os resultados, além do programa utilizado para análise ${ }^{2}$. A análise dos dados foi realizada pelo sistema de computador NTBS, com imagens topográficas de cada mama associadas ao aumento de calor, com pontuação de 0 a 10.

Existe um número elevado de protocolos, o que dificulta a utilização de apenas um padrão. Cada grupo de pesquisa adequa aparatos, câmeras, sala e aquisição de imagens e posições a sua realidade. Por essa diversidade, caraterísticas das coletas foram analisadas nas pesquisas e dispostas a seguir. 
Vários autores trabalharam na proposta РPJMT de identificação de câncer, embasadas na transferência de calor da lesão maligna e superfície 14; 22; 26; 32; 27; 28; 23; 33 ou no reconhecimento de padrões ${ }^{18 ; 32 ; 27 ; 5 ; 28}$.

As pesquisas apresentaram também uma preparação do paciente antes da coleta de imagens termográficas, com fatores como: não fazer uso do tabagismo e consumo de álcool e café, que podem causar alterações de pressão sanguínea e temperatura, aplicação de cosméticos, pomadas e atividade física. São conjuntos de instruções usadas, para não se realizar antes da coleta, até duas horas antes, para uma maior precisão da interpretação dos termogramas, são especificações dos seguintes autores: Shahari, Silva, 2015; Jesus De Guirro, 2017; Francis, 2014; Gárduno-Ramón, 2017; Morales-Cervantes, 2018 $30 ; 17 ; 8 ; 23 ; 29 ; 34$

Quanto ao ambiente, os vários autores recomendam uma aclimação do paciente. Cerca de $60,86 \%$ dos artigos, propõem colocar os pacientes para a realização de uma aclimatação, para gerar um equilíbrio térmico da paciente/ambiente 27; 23; 20; 21; 8; 2 . $15 ; 7 ; 30 ; 16 ; 3 ; 34 ; 26 ; 28 ; 29$. Assim, a paciente deve permanecer no ambiente do exame, despida da parte superior da roupa, por 10 a 15 minutos antes de iniciar a coleta ${ }^{15 ; 30 ; 29}$ a 15 20;23; $7 ; 28 ; 26$.

Os demais, cerca de $30,43 \%$ dos artigos presentes no portfólio não fizeram menção a aclimatação ou ao tempo de aclimatação ${ }^{14 ; 18 ; 31 ; 22 ; ~ 27 ; ~ 10 ; ~} 12$.

A temperatura para aclimatação apresentou-se em $78,26 \%$ das pesquisas, variando entre $20^{\circ} \mathrm{C}$ e $25^{\circ} \mathrm{C}^{14 ; 15 ; 22 ; 18 ; 35 ; 17 ; 26 ; 8}$ 20; 10; 11, com margem de alteração de $1^{\circ} \mathrm{C}$ $23 ; 15 ; 7$.

Quanto a posição que a paciente deve permanecer para aclimatação, em pé ou sentada, $30,43 \%$ dos artigos utilizaram a posição sentada com os braços apoiados na lateral da cadeira' 21,$27 ; 30 ; 15 ; 7 ; 29$ e 26,08\% ${ }^{17}$, 28; 34, 8; 11 na posição em pé com os braços atrás da cabeça. No restante das pesquisas a posição para a captação das imagens não foi mencionada.

Com relação a quantidade de imagens coletadas (nas coletas estáticas), apresentou-se uma média de 1 a 5 imagens de cada paciente. Aproximadamente 53,52\% dos artigos utilizaram apenas a posição frontal (1 imagem), e 43,47\% utilizaram imagens: frontal (1) mais lateral direita e lateral esquerda ( 2 imagens) e $34,78 \%$ com imagens frontal, lateral direita e esquerda e também imagens na postura oblíqua (2 imagens).

Dos 23 artigos analisados, apenas dois indicaram nos artigos, exatamente todo um conjunto de controle de substâncias na preparação das voluntárias para coleta das imagens termográficas (esse conjunto foi colocado conforme todas as características encontradas e apresentadas), horas antes da coleta como: controle da utilização de cafeína, cigarro, atividade física ou mesmo cremes na região; controle do ciclo menstrual e indicação da temperatura ambiente $\left(20^{\circ} \mathrm{C}\right.$ a $\left.25^{\circ} \mathrm{C}\right)$; além da aclimatação de 10 a 15 minutos do paciente; e utilização de biópsia, mamografia e/ou ultrassom para comparação 23; 17. Prosseguindo com um total de quatro artigos que indicaram a preparação antes da coleta, excetuando o ciclo menstrual8; 30; $29 ; 34$. Vale ressaltar que Jesus de Guirro (2017)9 e Garduño-Ramón (2017) 29 apresentaram sensibilidade e especificidade próximos a 100\%, embora, a diferença entre essas pesquisas e as outras, que citaram todo 0 conjunto preparatório, apresente-se principalmente, na utilização de câmeras com melhor tecnologia.

A discriminação do equipamento utilizado (Figura 5), mostrou o sistema FLIR como o mais utilizado, com $34,38 \%$ das câmeras. Embora, cerca de 34,39\% dos ar- 


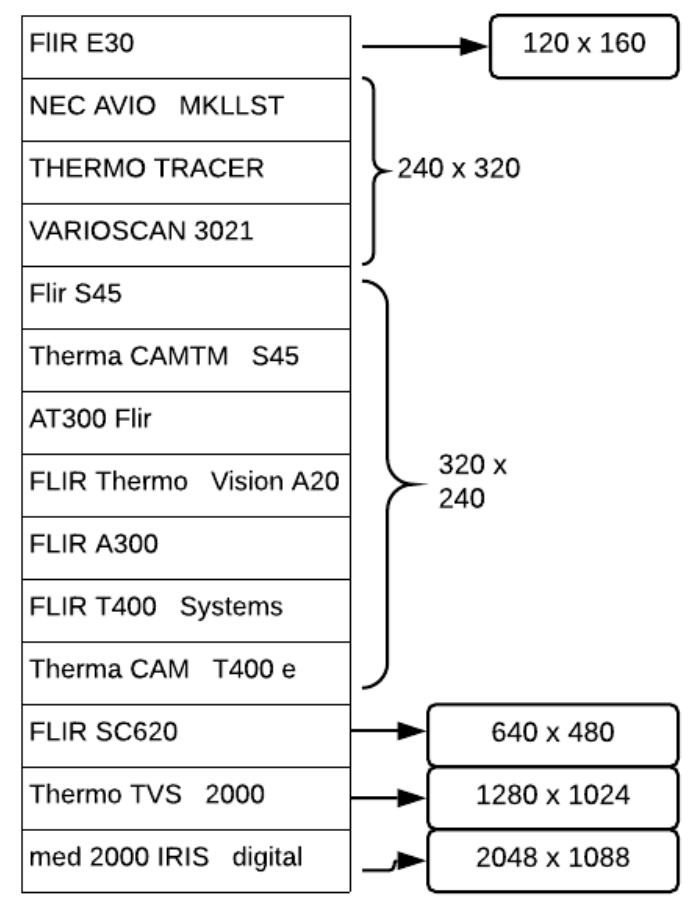

Figura 5- Câmeras utilizadas e pixel do equipamento

tigos não tenham mencionado a tecnologia empregada, as câmeras citadas apresentaram variações de resolução entre $160 \times 120(28 ; 32)$ a $1280 \times 1024^{3}$.

Nas análises do portfólio observou-se a divisão de dois protocolos já aceitos, embora não padronizados, quanto ao comportamento do corpo, que refere-se a transferência de calor, podendo ser: estático ou dinâmico.

Os protocolos estáticos levam em consideração o equilíbrio térmico com o ambiente. Os dinâmicos se referem à recuperação da temperatura do corpo após um estresse (térmico ou químico) ${ }^{12 ; 35 ; 36 ; ~ 5 ; ~} 17$.

Dos artigos encontrados, 47,82\% utilizaram o protocolo estático 20; 18; 27; 23; 31; 8 . 0 uso do protocolo dinâmico apresentou-se em $21,73 \%$ das pesquisas $21 ; 12 ; 15 ; 30 ; 17$.

A diversidade apresentou-se quanto aos meios utilizados para o estresse térmico, bem como o tempo do mesmo. Foram utilizados gelo na pontas dos dedos por 45/60 segundos $^{15}$, exposição da mama ao ventilador por: 2 a 6 minutos/ 3 a 4 minu- tos $^{27 ;} 23$ e 7 a 10 minutos $^{18}$, além de aplicação de álcool na região das mamas ${ }^{17}$.

\section{DIscussÃo}

Para a aquisição de imagens por termografia por infravermelho, algumas situações devem ser controladas para permitir a comparação com outros estudos e progresso de resultados, sendo elas:

- adequação do ambiente: a temperatura deve ser mantida entre $20^{\circ} \mathrm{C}$ a $25^{\circ} \mathrm{C}$, e constatou-se que a aclimatação tem resultados positivos para um melhor controle da temperatura do corpo. Assim, recomenda-se que o paciente deve permanecer de 10 a 15 minutos no espaço da coleta antes das imagens serem adquiridas. ${ }^{12 ; 9 ; 18}$.

- controle de substâncias na preparação do paciente: pesquisadores afirmam que, o que o paciente consome antes do exame, pode vir a alterar a taxa metabólica do corpo, causando variações nos termograma ${ }^{5 ;}$ 23; 17. Portanto, consumo de café, bem como tabagismo e consumo de álcool devem ser evitados até duas horas antes do exame, pois pode alterar a taxa metabólica do corpo levando às variações nos termogramas.

- aquisição de imagens: foi de senso comum a realização de menor movimento possível do paciente, independentemente da posição, apresentando-se como fator inerente a bons resultados.

- equipamentos utilizados: com relação aos resultados encontrados nos diversos estudos que utilizaram equipamentos de resoluções diferentes percebe-se melhores resultados em câmeras com maior número de pixels (acima de $160 \times 120$ ). Pesquisas que utilizaram câmeras de menores resoluções, apesar de não terem encontrado diferenças significativas de sensibilidade, afırmam que esses re- 
sultados na região da mama podem ser devido ao fato da região ser altamente irrigada. Morais et al (2016) ${ }^{26}$ em sua pesquisa, compararam duas câmeras, Sensor ThermaCam T400 FLIR E60, $320 \times 240$ pixels, e SAT-S160, $160 \times 120$ pixels, e constataram que ao aferir a temperatura de diferentes regiões do corpo humano (peito, olhos, mãos, pés (direito/ esquerdo), seios (direito/esquerdo), com termistor e câmera infravermelha, houve um erro sistemático de $2,2^{\circ} \mathrm{C}$ e $2,4^{\circ} \mathrm{C}$ para a câmera SAT-S160. Embora especificamente para os seios, a menor diferença foi de 0,0003 , enquanto a maior foi de 0,008 , diferente das outras regiões analisadas.

$\mathrm{Na}$ pesquisa de Raghavendra et al. (2016)3, (que utilizou a câmera de maior resolução do portfólio), câmera Thermo TVS 2000 MKLLST, px 1280x1024, encontrou-se uma sensibilidade de 96,66\%, especificidade de $100 \%$ e precisão de $98 \%$, utilizando 25 pacientes com CA maligno e 25 pacientes sem anormalidades na mama, com utilização de protocolo estático e nenhum procedimento de preparação de pacientes, apenas aclimatação.

Os protocolos de coleta utilizados, estático ou dinâmicos, apresentaram resultados de sensibilidade e especificidade médios de $90 \%$. No entanto a diferença de temperatura entre a região com tumor e a região normal se apresentou com maior nitidez nas imagens após o resfriamento do protocolo dinâmico, com um aumento de quase $30 \%$ de precisão, até mesmo para tumores pequenos. Os protocolos dinâmicos baseiam-se na exposição e estresse ao frio e acompanhamento do reaquecimento da região.

Usualmente, o protocolo dinâmico apresentou-se com duração do estresse ao frio com tempo máximo de 5 minutos, ou até alcançar temperaturas de resfriamento mais baixas e uniformes na região das mamas e axilas, para aumentar a visibilidade térmica do tumor.

Omranipouret al $(2016)^{15}$, realizaram a coleta de imagens de maneira estática e dinâmica, em 132 pacientes, sendo que o estresse ao frio foi realizado com teste de gelo, na região das mãos (extremidades), por 45-60 segundos, e com uma câmera de 640 x 480 px, resultando em uma sensibilidade combinada de ambos os métodos (MMG e TMG) de 96,2\%.

Francis et al $(2014)^{23}$ e Silva et al $(2015)^{17}$, também utilizaram ambas formas de coleta e constataram que protocolos dinâmicos são mais eficientes para destacar regiões quentes e vascularização na região das mamas, quando comparados a protocolos estáticos. Francis encontrou sensibilidade e especificidade de $81,82 \%$ e $100 \%$, respectivamente, utilizando sopro de ar fresco por 2 a 3 minutos, 22 pacientes com comprovação de câncer de mama por biópsia, objetivando a comprovação do mesmo por termografia. Silva et al $(2015)^{16}$, compararam quatro protocolos, sendo dois deles estáticos e dois dinâmicos. O primeiro estático, com aclimatação de 10 minutos e o segundo estático com aclimatação de 15 minutos, não observando diferenças entre os protocolos estáticos.

Dentre os protocolos dinâmicos: - ventilador direcionado para seios e axilas por dois minutos, gelo nas pontas dos dedos das mãos e álcool aplicado na região das mamas e axilas por 30 segundos, obteve-se resultados de maior visualização de vasos da região da mama e axilas, com o uso do ventilador, embora não tenha sido possível um resfriamento uniforme em apenas dois minutos (os autores sugerem aumentar o tempo para três a quatro minutos. 


\section{CONCLUSÃo}

Apesar dos diferentes protocolos, as pes-quisas apresentaram resultados promissores para o uso da termografia como técnica auxiliar no diagnóstico do câncer de mama.

Assim, um bom protocolo deve incluir uma aclimatação do paciente (entre 10 a 15 minutos), um ambiente com temperatura controlada de $20^{\circ} \mathrm{C}$ a $22^{\circ} \mathrm{C}$, câmeras com resoluções maiores que 160 × 120 pixels e aquisição de imagem com um proto-colo dinâmico.

As pesquisas tem avançado para o desenvol-vimento de programas e algoritmos para análise das imagens apresentadas, criando diferentes formas para extração das características e criação de classificadores para o diagnóstico entre tecidos sadios e tumorais.

\section{REFERÊNCIAS}

1. Inca. Instituto Nacional de Câncer José Alencar Gomes da Silva. Estimativa 2017: Incidência de Câncer no Brasil. Coordenação de Prevenção e Vigilância Rio de Janeiro: Inca, 2017.

2. Ng, EYK, Raghavendra, U. Na integraded index for breast cancer identification using histogram of oriented gradiente and kernel locality preserving projection features extracted from thermograms. Quantitative Infrared Thermography Journal, 2016. 13(2), 195-209. https://doi.org/10.1080/17686733.2016.1176734.

3. U. Raghavendra; U. Rajendra Acharya; E. Y. K. NG; JenHong Tan \& Gudigar. An integrated index for breast cancer identification using histogram of oriented gradi-ent and kernel locality preserving projection features extracted from thermograms. ISSN: 1768-6736 2116-7176; Journal homepage: http://www.tandfonline.com/loi/tqrt20.

4. Yadav, P, Jethani, V. Breast thermograms analysis for cancer detection using fetureextractionand data mining technique. Proceedings of the international conference on advances in information communication technology \& computing - AICTC16, 1-5, 2016. https:// doi.org/10.1145/2979779.2979866.

5. Kandlikar, SGP, Raghupathi, PAG. International Journal of heat and mass transfer infrared imaging technol-ogy for breast cancer detection - Current status, proto-cols and new directions. InternationalJournalofHeatand Mass Transfer, 108, 2303-2320, 2017. https://doi.org/10.1016/j. ijheatmasstransfer.2017.01.086.
6. Rassiwala, M, Mathur, P, farid, K. Evaluationof digital infra-redtermal imaging as naadjunctivescreeningmethod for breast carcinoma: a pilot-study. InternationalJournalofSurgery 12 (2014) 1439e1443.

7. Jesus de Guirro, RR, Vaz MMOLL, Neves, LMSN. Accuracyandreliabilityofinfreredthermography in as-ses smentofthebreastofwoamnaffectedby-cancer.JMedSyst (2017) 41: 87. DOI 10.1007/s10916-017-0730-7.

8. Gerassimova, E, Audit, B, Roux, SG.Wavelet-basedmu Itifractalanalysisofdynamicinfraredthermo-gramstoassist in earlybreastcancerdiagnosis, 2014.Frontiers in Physiology, 5 may, 1-11. https://doi.org/103389/ fphys.2014.00176.

9. Milosevic, M. Comparativeanalysisofbreastcancer de-tection in mammogramsandthermograms, 2015. Biomed. Eng.-Biomed. Tech. 2015; 60(1): 49-56.

10. Castro, FJ, Silva, SV, Motta, L. Um sistema para pré-procesamento de imagens térmicas e modelagem tridimensional aplicada à mastologia.Instituto de Computação-UFF, Departamento de Engenharia Mecânica UFPE, 3Coordenação de Informática-IFF. http://visual. ic.uff.br/,http://www.demec.ufpe.br.

11. Bezerra, LA, Oliveira, MM, Rolim, TL. Estimation of breast tumor termal properties using infrared images, 2013. Signal Processing 93 (2013) 2851-2863.

12. Yao, X, Wei, W, Wang, L. A comparison of mammography, and far-infrared thermography with pathological results in screening and early diagnosis of breast can-cer. Asian Biomedicine Vol. 8 No. 1 February 2014; 11-19. DOI: 10.5372/1905-7415.0801.257.

13. Vasconcelos, JH, Santos, WP, Lima, RCF. Analisys of methods of classification of breast thermographic images to determine their viability in the early breast cancer detection. IEEE Latin America Transactions, Vol. 16, N. 6, June 2018.

14. Omranipour, R, Kazemiah, A. Comparison of the accuracy of thermography and mammography in the detection of breast cancer. Breast Care2016;11: 260-264. DOI: $10.1159 / 000448347$

15. El-Sharkawy, Y, El-Sherif, A. High-performance nearinfrared imaging for breast cancer detection. Journal of Biomedical Optics 19(1), 016018 (January 2014).

16. Silva, LF, Oliveira, GOS. Silva, JB. Análise de séries temporaisde sinais térmicos da mama para detecção de anomalias. Researchgate, january 2014.https://www. researchgate.net/publication/277012853.

17. Antonini, S, Kolaric, D, Herceg, Z. Thermographic visualization of multicentric breast carcinoma.57th International Symposium ELMAR-2015, 28-30 Septem-ber 2015, Zadar, Croatia.

18. M. Etehadtavakol, C. Lucas, and S. Sadri, "Analysis of breast thermography using fractal dimension to establish possible difference between malignant and benign 


\section{PAN AMERICAN JOURNAL OF MEDICAL THERMOLOGY}

patterns", Journal of Health care Engineering. vol $1 \mathrm{~N}^{\circ}$ 1.pp. 27-43. 2010.

19. Acharya, UR, NG, EYK, Tan, JH. Thermography-ba sedbreastcancerdetectionusingtexturefeaturesandsupport vector machine .Journal Medical Systens. (2012) 36:1503-1510. DOI 10.1007/s10916-010-9611-z.

20. Araújo, RF, Alvarenga, L. a bibliometria na pesquisa científica da pós-graduação brasileira de 1987 a 2007. Encontros Bibli: revista eletrônica de biblioteconomia e ciência da informação, 16(31), 2011.

21. Venkataramani, K, Mestha, LK, Ramachandra, L. Semiautmed breast cancer tumor detection with thermographic vídeo imaging. 978-1-4244-9270-1/15/2015 IEEE.

22. Francis, SV, Sasikala, M, Saranya, S. Detction of breast abnormality from thermograms using curvelet transform based feature extraction. Journal Med Syst (2014) 38:23. DOI 10.1007/s10916-014-0023-3.

23. Ghayoumi, ZH, Haddadnia, J \&Montarezi, A. A model for diagnosing breast cancer our tissue from termal images using active contour and yapunov exponente. Iranian Journal of Public Health, 2016. 45(5), 657-669.

24. Mainiero, MB, Lourenço, A, Machoney, MC. ACR appropriateness criteria breast cancer screening, 2013. J Am Coll Radiol2013;10: 11-14.

25. Morais, KCC, Vargas, JVC. Na infrared based methodology for breast lesions screening. Infrared Physics \& Technology 76 (2016) 710-721.

26. Collet, AE, Guilfoyle, C, Gracely, EJ. Infrered imaging does not predict the presence of maligancy in patients with suspiciuous radiologic breast abnormalities, 2014. Wiley Periodicals, Inc., 1075-122X/14. The Breast Journal, Volume 20 Number 4, 2014 375-380.DOI: 10.1111/ tbj. 12273.

27. A.SP. Kiruhba. M. Andurajan. And B. Venkataramani. "Comparison pf PET-CT and Thermography with breast biopsy in evaluation of breast cancer: A case study". vol. 73. Pp. 115-125. July 2015.

28. M. A. Garduño-Ramon; S. G. Giovanna; L. A. Moráleshernadez; L. A. Osornio-Rios. Supportive Noninvasive Tool for the Diagnosis of Breast Cancer Using a Ther-mographic Camera as Sensor. Sensors 2017, 17, 497; doi:10.3390/ s17030497 www.mdpi.com/journal/sensors.

29. S. Shahari; A. Wakankar. Color Analysis of Thermograms for Breast Cancer Detection. Internacional Conference Instrumentation and Control (ICIC)College of Engineering Pune; Índia, 2015.

30. S. Godoy; D. A. Ramirez; S. A. Myers; G. V. Winckel; S. Krishna; M. Berwick; R. S. Padilla; P. Sem; S. Krishna. Dinamic Infrered imaging for skin Cancer screening. Infrered Phisics \& Psicology 70 (2015) 147-152.

31. Z. Zore; I. Filipóvic-Zore; M. Stanec; G. Batinjan; A. Matejcic. Associantion of clinical, histophatological and imnunohistochemical prognostic factors of invasive breast tumors and thermograpich findings. Journal homepage: www.elsevier.com/locate/infrared. 2014.

32. Li-Na Wu; Wen_Wung Kuo; Yuo-Show Tsai; J. Wang. The association of infrared imaging findings of the breast with prognosis in breast cancer patients: an observational cohort study. Wu et al. BMC Cancer (2016) 16:541 DOl 10.1186/s12885-016-2602-9.

33. A. Morales-Cervantes, E. S. Kolosovas-Machuca, E. Guevara; M. M. Reducindo; A. B. Bello Hernández; M. R. García; F. J. González. An Automated Method For The Evaluation Of Breast Cancer Using Infrared Thermography. EXCLI Journal 2018;17: 989-998 - ISSN 1611-2156.

34. Bhowmik, MK, Gogoi, UR. Designing of ground-truthannoted DBT-TU-JU breast thermogram database to-ward early abnormality prediction. IEEE Journal of Bio-medical and Health informatics, Vol. 22, No. 4, July 2018.

35. Z. Heidari; M. Dadgostar; Z. Einalou. Automatic Segmentation Of Breast Tissue Thermal Images. Biomedical Engineering: Applications, Basis and Communications, Vol. 30, No. 3 (2018) 1850024 (12 pages) DOl: $10.4015 /$ S1016237218500242. 